\title{
Mettre cette molécule au travail ?
}

Putting individual molecules to work?

\section{Christian Joachim}

\section{OpenEdition}

\section{Journals}

\section{Édition électronique}

URL : http://journals.openedition.org/philosophiascientiae/1809

DOI : 10.4000/philosophiascientiae.1809

ISSN : 1775-4283

Éditeur

Éditions Kimé

\section{Édition imprimée}

Date de publication : 18 février 2019

Pagination : 151-159

ISSN : 1281-2463

\section{Référence électronique}

Christian Joachim, « Mettre cette molécule au travail ? », Philosophia Scientiæ [En ligne], 23-1 | 2019,

mis en ligne le 01 janvier 2021, consulté le 31 mars 2021. URL : http://journals.openedition.org/ philosophiascientiae/1809; DOI : https://doi.org/10.4000/philosophiascientiae.1809 


\title{
Mettre cette molécule au travail ?
}

\author{
Christian Joachim \\ NanoScience Group, CEMES-CNRS, Toulouse (France)
}

Résumé : On retrace rapidement comment la molécule a pris son indépendance grâce notamment à l'invention du microscope à effet tunnel. Depuis le milieu des années 1970, son identité a changé : d'un quelconque indiscernable parmi une multitude (par exemple dans un nanomatériau) à une seule devant embarquer une petite unité de calcul ou délivrer une puissance motrice. Avec des molécule-machines complexes fonctionnant chacune indépendamment, on espère également taquiner la physique quantique autrement qu'en utilisant quelques photons ou un seul atome dans une cavité froide.

\begin{abstract}
We briefly retrace the story of how the Scanning Tunneling Microscope has allowed the molecule to establish its autonomy. Since the mid1970s, the identity of a molecule has changed from being any interchangeable unit in a multitude of identical entities (for example a single molecule within a nanomaterial) to being a specific individual molecule operating as a calculating unit or as an autonomous source of motive power. The complex autonomous molecule-machine promises to allow us to exploit properties predicted by quantum physics in a different way from the effects that depend on a few photons or a single atom in a cavity
\end{abstract}

Mettre une molécule au travail? Non pas collectivement dans un cristal ou une couche moléculaire, mais par elle-même, individuellement. Suivons le fonctionnement du molécule-interrupteur numéro 123985. Fait de quelques atomes, un de ses petits groupements chimiques bascule pour faire entrer une donnée numérique dans la molécule-calculateur faite de quelques milliers d'atomes. "C'est de l'électronique moléculaire, tout est dans les molécules » s'exclame en 1968 Brivaux dans le roman La Nuit des Temps de R. Barjavel [Barjavel 1968]. Par fabrication, le robot constructeur de cette électronique moléculaire a positionné la molécule-interrupteur à quelques 100 picomètres du fil électrique numéro 5674378. Ce fil atomique est d'un seul atome de section. Il a été construit atome par atome sur toute sa longueur à la surface d'un support 
isolant. Il permet d'apporter l'ordre de commutation de l'échelle du donneur d'ordre à la molécule-interrupteur 123985. Pour que la molécule comprenne cet ordre, il y aura également entre le donneur d'ordre et cette molécule, une conversion entre le très classique « tout ou rien » et de l'information quantique.

Ancrée sur le canal transmembranaire numéro 342675 à la surface d'un globule blanc, voici la molécule-moteur numéro 65435. Elle permet d'obstruer ce canal à volonté en utilisant un programme intégré pour pallier l'attaque d'un virus. Son énergie de rotation provient de l'agitation permanente des longues molécules constituant la membrane cellulaire. Cette énergie thermique est redressée par la structure électronique intime de cette molécule-moteur pour que son rotor tourne pas à pas et dans un seul sens afin d'ouvrir ou de fermer le canal [García-López, Chen et al. 2017].

En nous et d'un poids moléculaire beaucoup plus conséquent que les petites molécule-machines, des macromolécules biologiques travaillent à assurer le bon déroulement de nos fonctions vitales. Dès la fin des années 1970, quelques chercheurs se sont alors intéressés à l'encore plus petit et suffisamment stable qu'est une molécule d'une centaine d'atomes pour pouvoir utiliser ses ressources physiques intrinsèques afin de construire une petite unité de calcul de taille nanométrique [Aviram \& Ratner 1974]. Les premières molécule-roues ont été étudiées à l'unité sur une surface support à la fin des années 1990 [Gimzewski, Joachim et al. 1998].

Pour aller explorer ce monde matériel d'en bas où chaque molécule-machine doit fonctionner " comme une horloge », il faut un microscope adapté pour non seulement imager mais également toucher chaque molécule-machine. Pour ce faire, nous disposons maintenant de microscopes à champ proche. Parmi ces microscopes, le microscope à effet tunnel (STM) a été inventé au début des années 1980 [Binnig \& Rohrer 1986]. Il s'agissait d'abord d'aider à la poursuite de la miniaturisation des puces électroniques fabriquées à la surface d'un cristal semi-conducteur. Il permet, en effet, de réaliser des images de ce type de surface et des dopants atomiques avec une précision meilleure que $100 \mathrm{pm}$ ce que ne permet pas le microscope électronique (dit à champ lointain) qui fut inventé au début des années 1930 [Knoll \& Ruska 1932]. Avec son petit frère, le microscope à force atomique (AFM), ces microscopes à champ proche sont très vite devenus de formidables instruments d'exploration du nano-monde. La science des surfaces en a grandement bénéficié en permettant d'abord et de manière inégalée de déterminer la structure atomique exacte d'un grand nombre de surfaces. L'imagerie des molécules posées ou déposées sur ces surfaces a atteint un tel degré de précision qu'une nouvelle chimie de synthèse est en train de naître avec la synthèse chimique dite « sur-surface» de nouvelles molécules instables ou insolubles en solution en partant de précurseurs de faible poids moléculaire [Gourdon 2008].

Pour le nano-monde, et à la fin des années 1980, le STM est surtout devenu un formidable outil pour écrire atome par atome [Eigler \& Schweizer 1990], pour déplacer une seule molécule et par exemple la faire tourner en l'alimentant 
électroniquement en énergie. Construites dans les années 2010, les versions modernes à 4 têtes de ces microscopes permettent de nos jours les premières mesures de la conductance d'un fil atomique et bientôt d'une molécule-circuit électronique à multiples entrées-sorties [Kolmer, Olszowski et al. 2017].

Mais pourquoi mettre les molécules au travail une par une tout en respectant l'individualité de leur travail? On ne sait si les forgerons grecs du bronze de l'horloge d'Anticythère voulaient créer un marché économique des horloges astronomiques ou simplement exercer leur art. On sait par contre que la maîtrise du mouillage et l'invention des caractères mobiles également en bronze par Johannes Gutenberg visaient bien à créer un nouveau marché du document imprimé avant sa fameuse bible. Il en est de même pour la Pascaline de Blaise Pascal, même si ses premiers exemplaires en bois ne permettaient pas une grande diffusion commerciale. C'est également le cas pour l'invention de la triode à vide par Lee de Forrest et pour le transistor en semi-conducteur des laboratoires Bell à la fin des années 1940. La mise au travail d'une seule molécule avait également au départ un objectif technologique : la miniaturisation ultime des composants électroniques. Quand Ari Aviram \& Mark Ratner proposent à IBM d'utiliser une seule petite molécule pour un jour remplacer un rectificateur de courant fabriqué alors en silicium, ils ont en vue les marchés économiques futurs, en particulier pour la portabilité des machines électroniques et leur efficacité énergétique [Aviram \& Ratner 1974].

Avec l'invention de la microscopie à effet tunnel, sept ans plus tard, également chez IBM et avec force de capitaux, on pouvait donc s'attendre à ce que la technoscience donne toute sa puissance et que les moléculescalculateurs soient désormais entrées en production comme fruits de 40 ans de recherche intensive en électronique moléculaire. Or il n'en a rien été. Nous avons d'abord sous-estimé l'ampleur des défis technologiques nécessaires pour atteindre cet objectif. Nous avons surtout oublié qu'une poussée de fièvre technologique par envie d'exploration d'une limite, sous contraintes de santé publique, économique ou politique s'accompagne souvent d'un chambardement scientifique. Au cas où et par anticipation le mot «nanoscience » est quand même apparu dans les années 1990. Peut-être ce mot a-t-il été forgé trop rapidement.

Orpheline dans sa définition première « sciences à l'échelle du nanomètre », la nanoscience est alors assez dépourvue de résultats scientifiques. Pas de nouvelle loi, et trop peu de nouveaux objets d'étude. Elle est donc appelée en renfort pour rassembler des champs technoscientifiques loin de sa définition initiale et ceci sans avoir besoin de l'indépendance moléculaire [Joachim 2005]. Première déviation au milieu des années 1990 : la physique mésoscopique, intermédiaire entre la microphysique et la nano-physique. Deuxième déviation : les nanomatériaux vers la fin des années 1990. Et enfin troisième déviation vers les technologies quantiques aux alentours des années 2010. En effet et pour le temps présent, on s'en sort très bien technologiquement en assemblant un grand nombre de molécules (ou de nanoparticules) pour créer des nanomatériaux aux propriétés nouvelles et donc des produits nouveaux. 
Ce foisonnement d'idées à base de nanomatériaux a totalement occulté la prise d'indépendance moléculaire. Le champ des nanosciences et nanotechnologies est alors progressivement devenu le lieu de la physique mésoscopique et des matériaux nano-structurés On s'en sort également très bien en émulant les comportements quantiques avec des systèmes mésoscopiques. L'espoir de la micro-électronique a maintenant basculé de l'électronique moléculaire vers les calculateurs quantiques [Nielsen \& Chuang 2002]. Dans un futur proche, ceuxci pourraient battre en performances nos bons vieux processeurs en silicium. Ici encore, point n'est besoin d'une seule molécule pour faire le travail.

Ces deux vagues technoscientifiques des nanomatériaux, puis des calculateurs quantiques, ont finalement libéré la créativité scientifique en nanosciences de son carcan technologique. Ce qui semblait être un double péché de naissance avec comme objectif final le futur du marché économique de la microélectronique : électronique moléculaire et invention de la microscopie à effet tunnel est en passe d'être oublié. Il est redevenu possible d'essayer de faire travailler une seule et toujours la même molécule, objet d'étude par excellence de la nanoscience, sans avoir à produire pour demain la puce moléculaire qui va sauver la loi de Moore. Prenons deux exemples de cette libération : le fil moléculaire et la molécule-moteur.

Les fils moléculaires devaient être à la base de l'architecture des processeurs de l'électronique moléculaire [Joachim, Gimzewski et al. 2000]. Chaque fil moléculaire devait réaliser l'interconnexion entre deux composants moléculaires dans une sorte de copie minuscule des circuits électroniques des puces de la micro-électronique actuelle. Il a été très difficile de mesurer la conductance électronique d'un seul et unique fil moléculaire tout en connaissant parfaitement sa composition chimique et sa structure. Mais à force de prouesses expérimentales, les premières mesures ont été effectuées à la fin des années 2000, grâce notamment à la nouvelle chimie « sur-surface » et à l'amélioration de la stabilité du microscope à effet tunnel. Confirmation expérimentale : l'intensité du courant électronique passant au travers d'un fil moléculaire décroît exponentiellement avec la longueur de ce fil. Le taux de cette décroissance est même très grand, à peu près un ordre de grandeur par nanomètre de longueur de fil [Lafferentz, Ample et al. 2009]. Il devient alors difficile de construire un circuit électronique complexe, c'est-à-dire très étendu dans l'espace. L'intérêt technologique pour ces fils moléculaires (et le petit frère : le fil de section atomique stabilisé à la surface d'un isolant) a donc fortement diminué entre la fin des années 1990 et le début des années 2010. On s'est également aperçu que les bonnes vieilles lois des mailles et des nœuds de Gustav Kirchhoff n'étaient plus valables dans un circuit moléculaire. Avec tout ceci l'électronique moléculaire semble bien mal engagée pour révolutionner la micro-électronique. De plus, le transistor en semi-conducteur, composant élémentaire essentiel de toute puce électronique continue à être miniaturisé pour atteindre de nos jours et en laboratoire, une longueur de la zone active de l'ordre de $5 \mathrm{~nm}$. La seule molécule-transistor ayant montré un gain expérimentalement demande une jonction métal-molécule-métal de $2 \mathrm{~nm}$ 
de long [Joachim \& Gimzewski 1997]. La microélectronique rejoint donc l'électronique moléculaire. Premier marqueur historique majeur de l'entrée en nanosciences, l'électronique moléculaire version électronique du futur semble donc vouée à disparaître. Elle est avalée à la fois par le rouleau compresseur de la micro-électronique devenue au fil des années « nanoélectronique » et par la technologie nouvelle des calculateurs quantiques dont les bases théoriques ont été jetées par Richard Feynman et David Deutch en 1984 [Feynman 1984], soit dix années après l'article théorique de Aviram \& Ratner sur la molécule-diode.

C'est justement la confrontation scientifique et technologique entre l'atterrissage réaliste de l'électronique moléculaire et l'envolée spectaculaire des études et sources de financement sur les calculateurs quantiques qui a entraîné une petite renaissance pour le fil moléculaire. Et si l'ingénierie quantique permettait de concevoir de nouveaux fils moléculaires «quantiques » sans décroissance exponentielle de leur conductance électronique? La première publication ouvrant cette voie est parue en 2015 [Nacci, Ample et al. 2015]. De cette hybridation entre l'électronique moléculaire et l'ingénierie quantique est en train de naître un nouveau champ de recherche qui forge vraiment une partie de la nanoscience. La nouvelle chimie « sur-surface » est ici très utile car les premiers fils moléculaires de cette nouvelle voie ne peuvent pas ici être synthétisés en solution. Ils sont alors synthétisés en un très petit nombre à la surface d'un métal par diffusion bidimensionnelle de monomères. Ils sont ensuite choisis un par un par la pointe du microscope à effet tunnel après avoir obtenu leur image par la même technique. La réactivité chimique latérale de ces monomères est activée thermiquement. Autre nouveauté, la longueur de ces fils peut atteindre de $50 \mathrm{~nm}$ à $100 \mathrm{~nm}$. Les constructeurs de STM développent donc maintenant des instruments à plusieurs têtes pour pouvoir mesurer avec au moins deux pointes, la conductance de ces nouveaux fils moléculaires quantiques. En nanoscience, on rafraîchit même ici ses connaissances en physique quantique en ressortant la théorie des bandes à valeurs complexes, une théorie formulée à la fin des années 1950 par Walter Kohn, prix Nobel de chimie en 1998 [Kohn 1959]. Cette théorie longtemps considérée comme un petit joyau mathématique sans trop d'utilité est maintenant utilisée dans le domaine des fils moléculaires quantiques, pour donner une courbe universelle du taux de décroissance tunnel en fonction des caractéristiques de la structure électronique des fils moléculaires [Nacci, Ample et al. 2015]. On espère également observer une signature vibrationnelle des électrons tunnel transférés le long du fil moléculaire dans une sorte de potentiométrie tunnel à 3 pointes afin de taquiner les ressorts des comportements quantiques. L'effet tunnel résistera-t-il quand on observe un courant tunnel de l'intérieur de la barrière? On a même conçu une petite molécule ampèremètre à insérer le long du fil moléculaire pour compter en moyenne le nombre d'électrons passant par effet tunnel au travers de ce fil.

La molécule-moteur a eu deux naissances expérimentales. La première vient d'une longue tradition de la chimie moléculaire et supramoléculaire à vouloir bien maîtriser le mouvement des molécules en solution, prolongement 
des intuitions du biochimiste Paul D. Boyer, prix Nobel de chimie en 1997, qui voyait au milieu des années 1960 dans les changements de conformation de protéines une explication par mécanique moléculaire des mécanismes élémentaires du vivant. Le récent prix Nobel de chimie 2016, attribué à JeanPierre Sauvage, James Frazer Stoddart et Bernard Feringa, est une illustration parfaite de cette première naissance vers le milieu des années 1980. Sauvage et Stoddart s'intéressaient alors en particulier à des molécules entrelacées et au mouvement relatif des anneaux en fonction de différentes stimulations chimiques ou optiques [Livoreil, Dietrich-Buchecker et al. 1994].

La deuxième naissance est plus récente et découle de l'invention du STM. Suite à nos études sur les mécanismes physiques produisant le contraste intramoléculaire dans l'image d'une molécule obtenue par microscopie à effet tunnel, nous nous sommes aperçus en 1998 qu'il était possible à volonté de mettre en rotation une seule de ces molécules, d'un diamètre de $1,2 \mathrm{~nm}$ [Gimzewski, Joachim et al. 1998]. Bel exemple de nanoscience! Il nous a fallu alors comprendre les lois de ce mouvement de rotation moléculaire. Il peut être déclenché soit par une petite impulsion électrique appliquée à un endroit précis sur la molécule choisie, soit en déplaçant justement l'axe de rotation de cette molécule de $0,255 \mathrm{~nm}$ par manipulation moléculaire avec la pointe du microscope. Nous nous sommes alors posé un grand nombre de questions : une molécule-rotor peut-elle tourner dans un seul sens [Perera, Ample et al. 2013] ? Comment contrôler son sens de rotation? À quelle vitesse tourne-t-elle sur sa surface support? Y a-t-il de la friction électronique et/ou mécanique entre cette molécule-rotor et la surface support? Une molécule peut-elle avoir une puissance motrice [Ohmann, Meyer et al. 2015]? Quels sont les canaux permettant à l'énergie d'entrer pour fournir la puissance nécessaire à ce travail ? Si plusieurs de ces roues moléculaires sont montées sur un châssis également moléculaire de quelques nanomètres de longueur, les roues peuvent-elles tourner? Avons-nous assez de place pour monter une moléculemoteur sur le châssis d'un tel nano-véhicule? Peut-il y avoir transmission d'un mouvement de rotation le long d'une longue chaine de molécule-engrenage construite molécule par molécule?

De ces interrogations post-1998 est né le champ de la nano-mécanique moléculaire qui vient également de plein droit remplir le champ de la nanoscience. Chimistes de synthèse et chimistes théoriciens essayent maintenant de concevoir des molécule-moteurs ayant un minimum de puissance motrice. Les physiciens des surfaces imaginent des protocoles expérimentaux afin de mesurer cette puissance en utilisant les nouveaux microscopes à plusieurs pointes, une pointe de microscope à effet tunnel pour alimenter en énergie la molécule-moteur et une pointe de microscope à force atomique pour mesurer la puissance fournie. Certains expérimentateurs ont même commencé à s'interroger sur les limites de la puissance motrice de la plus petite machine matérielle possible avec un piston constitué d'un seul atome en utilisant la technologie des atomes froids. Les chimistes théoriciens et leurs collègues physiciens commencent à établir les lois du mouvement du rotor de ces 
molécule-moteurs et prédire leur rendement [Echeverria, Monturet et al. 2014]. Le STM a même permis de construire une première crémaillère moléculaire et la construction d'un train d'engrenage est en cours. Chaque moléculeengrenage a un diamètre compris entre 1 et $2 \mathrm{~nm}, 6$ dents moléculaires et son axe de rotation est constitué d'un seul atome métallique.

Voici donc les débuts de la nanoscience. L'objet d'étude est une seule molécule, toujours la même. Nous allons la mettre en situation d'étude puis la faire travailler, calculer ou transmettre de l'information. Nos nouveaux protocoles expérimentaux demandent soit une surface, soit un piège optique pour maintenir la molécule-dispositif ou la molécule-machine choisie en situation. Ils requièrent l'invention de nouveaux modes de communication entre la molécule et l'expérimentateur, le donneur d'ordre ou fournisseur d'information. Une molécule apprécie l'échange d'information par voie quantique. Mais nous ne savons pas trop parler le quantique d'où le constat qu'en moléculemécanique, les études sur surface sont les plus développées, car les mouvements mécaniques sont alors presque classiques [Echeverria, Monturet et al. 2014]. Il s'agit maintenant d'optimiser la structure électronique intrinsèque de la molécule-machine pour que, par exemple, une molécule-moteur produise du travail avec un bon rendement. Il s'agit maintenant d'augmenter la puissance de calcul d'une molécule en minimisant son nombre d'atomes. Nous en sommes actuellement à seulement 30 atomes de carbone et deux atomes d'or pour une molécule porte logique booléenne [Soe, Manzano et al. 2011]. Il s'agit maintenant de jouer avec l'effet tunnel sur de grandes distances intramoléculaires pour qu'un fil moléculaire devienne un véritable canal quantique de transmission d'information. On s'approche enfin d'une question post-quantique : pourquoi le principe de superposition agrémenté du postulat de Born semble être le principe de fonctionnement d'une molécule-moteur et semble gouverner la puissance calcul d'une molécule à calculer [Joachim 2006] ? Quelque part en nanoscience, peut-on craquer la mécanique quantique en poussant le développement des molécule-machines?

\section{Bibliographie}

Aviram, Arieh \& Ratner, Mark A. [1974], Molecular rectifiers, Chemical Physics Letters, 29(2), 277 -283, doi : 10.1016/0009-2614(74)85031-1.

Barjavel, René [1968], La Nuit des temps, Paris : Presses de la Cité.

Binnig, Gerd \& Rohrer, Heinrich [1986], Scanning tunneling microscopy, IBM Journal of Research and Development, 30(4), 355.

Echeverria, Jorge, Monturet, Serge et al. [2014], One-way rotation of a molecule-rotor driven by a shot noise, Nanoscale, 6, 2793-2799, doi : 10 . 1039/C3NR05814J. 
Eigler, Donald M. \& Schweizer, Erhard K. [1990], Positioning single atoms with a scanning tunnelling microscope, Nature, 344, 524-526, doi : 10.1038/ $344524 \mathrm{a} 0$.

Feynman, Richard P. [1984], Quantum-mechanical computers (A), Journal of the Optical Society of America B Optical Physics, 1, 464.

García-López, Victor, Chen, Fang et al. [2017], Molecular machines open cell membranes, Nature, 548, 567-572, doi : 10.1038/nature23657.

Gimzewski, Jim K., JoAChim, Christian et al. [1998], Rotation of a single molecule within a supramolecular bearing, Science, 281(5376), 531-533, doi : 10.1126/science.281.5376.531.

Gourdon, André [2008], On-surface covalent coupling in ultrahigh vacuum, Angewandte Chemie International Edition, 47(37), 6950-6953, doi : 10. 1002/anie.200802229.

JoAChim, Christian [2005], To be nano or not to be nano?, Nature Materials, 4, 107-109, doi : 10.1038/nmat1319.

[2006], The driving power of the quantum superposition principle for molecule-machines, Journal of Physics : Condensed Matter, 18(33), 19351942, doi : 10.1088/0953-8984/18/33/S11.

JoAchim, Christian \& Gimzewski, Jim K. [1997], An electromechanical amplifier using a single molecule, Chemical Physics Letters, 265(3), 353357, doi : 10.1016/S0009-2614(97)00014-6.

JoAChim, Christian, Gimzewski, Jim K. et al. [2000], Electronics using hybridmolecular and mono-molecular devices, Nature, 408, 541-548, doi : 10.1038/ 35046000 .

Knoll, Max \& Ruska, Ernst [1932], Das Elektronenmikroskop, Zeitschrift für Physik, 78(5), 318-339, doi : 10.1007/BF01342199.

Koнn, Walter [1959], Analytic properties of Bloch waves and Wannier functions, Physical Review, 115, 809-821, doi : 10.1103/PhysRev.115.809.

Kolmer, Marek, Olszowski, Piotr et al. [2017], Two-probe STM experiments at the atomic level, Journal of Physics : Condensed Matter, 29(44), 444 004, doi : 10.1088/1361-648X/aa8a05.

Lafferentz, Leif, Ample, Francisco et al. [2009], Conductance of a single conjugated polymer as a continuous function of its length, Science, 323(5918), 1193-1197, doi : 10.1126/science.1168255.

Livoreil, Aude, Dietrich-Buchecker, Christiane O. et al. [1994], Electrochemically triggered swinging of a [2]-catenate, Journal of the American Chemical Society, 116(20), 9399-9400, doi : 10.1021/ja00099a095. 
NaCCI, Christophe, Ample, Francisco et al. [2015], Conductance of a single flexible molecular wire composed of alternating donor and acceptor units, Nature Communications, 6, 7397-7404, doi : 10.1038/ncomms8397.

Nielsen, Michael \& ChuAng, Isaac [2002], Quantum Computation and Quantum information, Cambridge : Cambridge University Press.

Ohmann, Robin, Meyer, Jörg et al. [2015], Supramolecular rotor and translator at work : On-surface movement of single atoms, ACS Nano, 9(8), 8394-8400, doi : 10.1021/acsnano.5b03131.

Perera, Uduwanage Gayani E., Ample, Francisco et al. [2013], Controlled clockwise and anticlockwise rotational switching of a molecular motor, Nature Nanotechnology, 8, 46-51, doi : 10.1038/nnano.2012.218.

Soe, We-Hyo, Manzano, Carlos et al. [2011], Manipulating molecular quantum states with classical metal atom inputs : Demonstration of a single molecule NOR logic gate, ACS Nano, 5(2), 1436-1440, doi : 10.1021/ nn1033899. 